\title{
Comportamiento social y reproductivo del roedor subterráneo solitario Ctenomys talarum (Rodentia: Ctenomyidae) en condiciones de semicautiverio
}

\author{
Social and reproductive behavior of the subterranean solitary rodent Ctenomys \\ talarum (Rodentia: Ctenomyidae) in a seminatural enclosure
}

\author{
ROXANA R. ZENUTO ${ }^{1,2}$, ALDO I. VASSALLO ${ }^{1,2} \&$ CRISTINA BUSCH $^{1}$ \\ ${ }^{1}$ Departamento de Biología, Facultad de Ciencias Exactas y Naturales, Universidad Nacional de Mar del \\ Plata, Casilla de Correo 1245, (7600) Mar del Plata, Argentina; \\ e-mail: rzenuto@mdp.edu.ar \\ ${ }^{2}$ Comisión Nacional de Investigaciones Científicas y Técnicas
}

\section{RESUMEN}

Se estudió el comportamiento social y reproductivo en grupos de individuos del roedor subterráneo Ctenomys talarum (Thomas, 1898) en condiciones seminaturales, que reflejaron la complejidad de las cuevas de la especie así como también la relación de sexos típica de poblaciones naturales. Los datos sugieren que las interacciones entre los individuos son mucho más variadas y complejas que lo esperado para una especie solitaria y territorial. Diferentes canales de comunicación -auditivo, táctil y olfativo- fueron importantes para el establecimiento y mantenimiento de territorios y durante el cortejo y la cópula. Se observaron diferencias en el comportamiento social entre individuos de poblaciones naturales con diferentes características ecológicas. Individuos pertenecientes a la población de Mar de Cobo, que presenta una alta densidad y una proporción de sexos desviada a favor de las hembras, establecieron jerarquías de dominancia entre los machos, lo que determinó el acceso preferencial por parte del macho dominante a varias hembras. La observación de daño físico (heridas) fue común entre machos de la población de Mar de Cobo. En contraste, la agresión entre machos de la población de Necochea, que presenta una baja densidad y una proporción de sexos balanceada, usualmente no resultó en daño físico y no se detectó una jerarquía de dominancia; los machos cortejaron a las hembras aún en presencia de otros machos.

Palabras clave: comportamiento social, reproducción, roedores subterráneos, cautiverio, Ctenomys talarum.

\begin{abstract}
The social and reproductive behavior of the subterranean rodent Ctenomys talarum (Thomas, 1898) was studied in a seminatural enclosure resembling both the complexity of the natural burrow system and the sex ratio observed in wild populations. Contrary to expectations based on their solitariness and their rather simple social system, the interactions among individuals of this species were diverse and complex. Auditory, tactile and chemical communication were of importance during territory guarding, courtship and copulatory behavior. Individuals from different populations differing in ecological attributes behaved in substantially different ways. Males from Mar de Cobo, a population characterized by a high density and a skewed sex ratio favouring females, behaved aggressively, which resulted in frequent wounding. Males from Mar de Cobo established a dominance hierarchy among them, which preceded courtship and, eventually, resulted in the dominant male monopolizing mating activity. On the contrary, males from Necochea, a population showing low density and an even sex ratio, behaved less aggressively; dominance relationships were absent, and males performed courtship behaviors in the presence of other males.
\end{abstract}

Key words: social behavior, reproduction, subterranean rodents, captivity, Ctenomys talarum.

\section{INTRODUCCIÓN}

Los estudios que tratan comportamiento social representan una parte importante de los esfuerzos destinados a comprender la biología de los roedores subterráneos. Aún en especies en las cuales cada adulto habita una madriguera, los individuos deben en algún momento contactarse para repro- ducirse, lo cual provee una oportunidad para la evolución de interacciones sociales complejas. Tales interacciones pueden afectar caracteres fenotípicos tales como la demografía, el éxito reproductivo y la estructura genético-poblacional, por lo que el comportamiento social puede tener consecuencias evolutivas a nivel poblacional (Lacey 2000). 
Los roedores subterráneos conforman ocho familias, alrededor de 30 géneros y varios cientos de especies reconocidas ocupando vastas regiones del Viejo y Nuevo Mundo (Nevo 1979). Los sistemas sociales de este grupo de roedores permanecen pobremente conocidos, lo cual dificulta proponer hipótesis sobre el posible rol del ambiente subterráneo en modelarlos (Lacey 2000). Esto puede atribuirse a problemas logísticos; los hábitos ocultos de estos organismos -que desempeñan casi la totalidad de sus actividades en cuevas cerradas bajo tierra- impiden la visualización directa de las interacciones entre conespecíficos.

Los métodos alternativos que permiten obtener información respecto de interacciones sociales en roedores subterráneos solitarios incluyen el trampeo de extracción total y el de marcado y recaptura (Hansen \& Millar 1959, Vaughan 1962, Patton \& Feder 1981, Busch et al. 1989, Daly \& Patton 1990, Malizia et al. 1995, Lacey et al. 1998, Zenuto \& Busch 1998). El aporte de la radiotelemetría permite conocer más detalles respecto de movimientos de los individuos (Bandoli 1987, Zuri \& Terkel 1996, Lacey et al. 1997, 1998) y su evolución en el tiempo (reconocer si son solitarios o sociales, la ubicación relativa de sexos en el terreno, quiénes se dispersan, los patrones temporales de actividad, los cambios en las afinidades espaciales entre individuos). Un aspecto adicional corresponde al aporte de análisis moleculares que han comenzado a revelar relaciones de parentesco y estructura reproductiva de algunas especies (Patton \& Feder 1981, Patton \& Smith 1993, Zenuto et al. 1999a). El estudio de animales en condiciones de cautiverio permite abordar aspectos del comportamiento social difíciles de observar en estudios de campo. La calidad de estos estudios ha aumentado como resultado de la implementación de condiciones que disminuyen el stress y la agresión entre los individuos mediante el aumento del tamaño y complejidad estructural de los encierros, lo que se traduce en que los individuos no se encuentran forzados a interactuar constantemente (Shanas et al. 1995, Gazit et al. 1996, Gazit \& Terkel 1998, 2000, Zenuto et al. 2001).

Ctenomys talarum es un roedor subterráneo herbívoro que habita sistemas de galerías subterráneas. Ambos sexos en todas las edades (excepto las crías hasta el destete) son sedentarios y presentan territorialidad individual (Busch et al. 1989). Dos poblaciones de esta especie muestran diferencias en caracteres demográficos (Busch et al. 1989, Malizia 1994, Malizia et al. 1995, Malizia \& Busch 1997). La población de Mar de Cobo se caracteriza por altas densidades (65 ind $\mathrm{ha}^{-1}$ ), proporciones sexuales desviadas hacia las hem- bras en adultos y dispersión con predominio de machos. La población de Necochea presenta densidades menores (13 ind ha ${ }^{-1}$ ), proporciones sexuales no desviadas, y dispersantes inmaduros de ambos sexos. En ambas localidades, los individuos muestran un patrón de establecimiento de madrigueras donde cada macho se encuentra rodeado por más de una hembra.

Desde Emlen \& Oring (1977), el potencial ambiental para la monopolización de apareamientos es reconocido como un importante modelador de los sistemas reproductivos. Así, resulta posible encontrar diferencias en el sistema de apareamiento de poblaciones de la misma especie sujetas a diferentes condiciones ambientales (recursos) o de densidad. Para los mamíferos, se predice que a mayor potencial de monopolización múltiple de parejas, más intensa será la selección intrasexual en tamaño corporal. Además, debido a que la selección sobre el tamaño de los testículos es consecuencia de la frecuencia copulatoria (Short 1977), se esperan pequeños tamaños testiculares en machos pertenecientes a sistemas de apareamiento de "un solo macho" (monogamia, poliginia extrema) y tamaños mayores en los pertenecientes a sistemas de apareamiento de "varios machos" (promiscuidad, poliginia con más de un macho por grupo reproductivo) (Kenagy \& Trombulak 1986). Una alta producción de espermatozoides sería efectiva para incrementar el éxito de apareamiento cuando el esperma de diferentes machos compite en el tracto genital femenino (Ågren 1990). Así, los machos de una misma población podrían modular su producción de espermatozoides conforme a la probabilidad de ocurrencia de competencia de esperma (Baker and Bellis 1989). Si bien se ha demostrado mediante marcadores moleculares que ambas poblaciones de $C$. talarum son poligínicas (Zenuto et al. 1999a), otras fuentes de evidencia sugieren la existencia de distintos grados de poliginia, siendo ésta más extrema en la población de Mar de Cobo. Evidencias de una competencia más acentuada entre machos por apareamientos provienen de (a) un dimorfismo sexual (siendo los machos de mayor tamaño que las hembras) mayor en Mar de Cobo comparado con Necochea (Zenuto et al. 1999b), (b) un menor tamaño relativo de los testículos en machos de Mar de Cobo (Zenuto et al. 1999b), y (c) una baja concentración espermática en los epidídimos en machos de ambas poblaciones, lo cual concuerda con el tamaño pequeño de los testículos. Sin embargo, los machos de Mar de Cobo presentaron números espermáticos bajos y similares mientras la producción espermática de los machos de Necochea es mayor y más variable (Zenuto 1999). Estos resultados sugieren que en 
Mar de Cobo los machos monopolizan el acceso a varias hembras mientras que en Necochea el acceso a las hembras por parte de los machos sería más equitativo.

Con objeto de examinar las predicciones anteriores, el presente trabajo describe y cuantifica la naturaleza y diversidad de interacciones sociales entre conespecíficos de la especie C. talarum. En particular, se investigó si las diferencias poblacionales mencionadas se correlacionan con diferencias en la habilidad de los machos para acceder a las hembras, así como también en el comportamiento de cortejo. Se utilizó una metodología que ha permitido la primera reproducción en cautiverio para la especie (Zenuto et al. 2001) y que, a diferencia de otros sistemas utilizados en especies de similares características (Gazit et al. 1996, Borroni \& Capanna 1999), permitió la interacción de más de un par de individuos.

\section{MATERIALES Y MÉTODOS}

\section{Sistema de túneles artificiales}

Se utilizó un sistema de cuevas artificiales construidas con tubos de PVC blanco y vidrio en la parte superior a fin de posibilitar la observación de los animales. Cada cueva artificial compren- dió un recorrido de 3,5 m de túnel (100 mm de diámetro), una caja de recursos y una cámara de crianza (nido). Este diseño representa, en menor escala, la complejidad estructural descripta para los sistemas de cuevas en el campo, las cuales constan de un túnel principal de $6 \mathrm{~m}$ de longitud, un promedio de ocho ramificaciones y un único nido (Antinuchi \& Busch 1992). Todas las cuevas (cinco para el caso de Mar de Cobo, cuatro para Necochea) fueron conectadas a un espacio de uso común que consistió en una caja plástica transparente de 50 × 35 × $28 \mathrm{~cm}$ (Fig. 1). Las conexiones a esta caja habitualmente permanecieron cerradas y sólo fueron abiertas durante el tiempo en que se llevaron a cabo las observaciones, de forma tal que los animales pudieran interactuar libremente. La caja de recursos fue provista de una tapa con alambre tejido, a fin de otorgar la apariencia de "condición externa", dado que los tucotucos recolectan la vegetación en superficie accediendo por las bocas de alimentación (Busch et al. 2000). El espacio de uso común también fue provisto de una tapa con alambre tejido ya que en la naturaleza las cuevas individuales no muestran conexiones subterráneas (Antinuchi \& Busch 1992). Por otro lado, los túneles y las cajas nido se mantuvieron completamente cerradas representando así la condición natural.

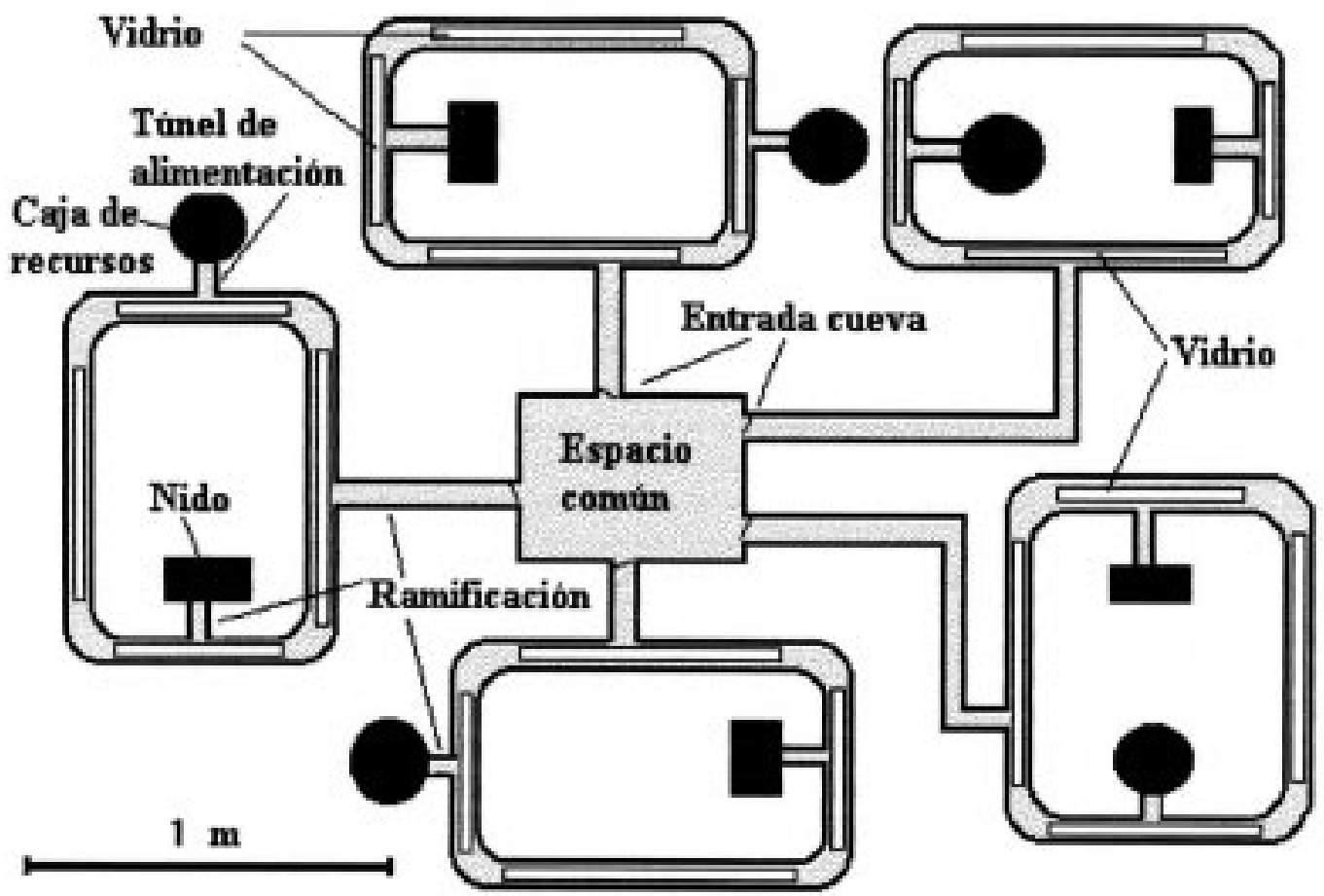

Fig. 1: Esquema del sistema de cuevas utilizado para el estudio de comportamiento social y reproductivo en el roedor subterráneo Ctenomys talarum de la localidad de Mar de Cobo.

Experimental enclosure used to study social and reproductive behavior in the subterranean rodent Ctenomys talarum from Mar de Cobo locality. 


\section{Animales}

Los individuos utilizados correspondieron a animales adultos capturados en las localidades de

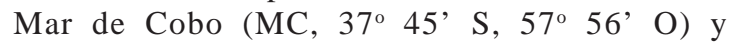
Necochea (N, 38 33' S, 58 $45^{\circ}$ O), Provincia de Buenos Aires, Argentina, con trampas de captura viva realizadas con tubos de PVC (diámetro: 110 mm, longitud: $350 \mathrm{~mm}$ ). La mismas fueron ubicadas en las entradas de las cuevas y constan de una puerta que se cierra al momento en que un individuo presiona un piso falso, mientras que el extremo opuesto posee una malla de alambre fija. Una vez transportados al laboratorio, dos machos y tres hembras fueron dispuestos en forma individual en cada cueva artificial para el caso de Mar de Cobo, de acuerdo a la proporción sexual encontrada en la población natural (1 macho: 1,63 hembras, Busch et al. 1989). Utilizando el mismo criterio, se dispusieron cuatro individuos (dos machos y dos hembras) capturados en Necochea (proporción sexual = 1 macho: 1 hembra, Malizia \& Busch 1997) en sus respectivas cuevas. Dado que resulta imposible conocer el estado reproductivo de las hembras utilizando señales externas, las mismas fueron capturadas en estado de preñez, analizándose su interacción con otros individuos del sistema a partir de la etapa de celo post-parto. Durante el transcurso del presente estudio, un macho de MC fue reemplazado al igual que un macho y una hembra de $\mathrm{N}$, por lo que el número de individuos analizados en ambos casos fue de seis (tres machos y tres hembras). El individuo de MC fue retirado del sistema debido a que resultó severamente herido durante las interacciones agresivas; a partir de allí se aseguró su recuperación física en aislamiento y posteriormente fue liberado en su ambiente natural. En el caso de $\mathrm{N}$, los reemplazos de individuos ocurrieron como consecuencia de muerte natural y escape, respectivamente. El reconocimiento individual de los animales fue posible mediante el uso de marcas plásticas de diferentes colores $(10 \mathrm{~mm}$ de diámetro) adheridas al lomo de los individuos con pegamento de cianocrilato.

\section{Mantenimiento y dieta}

Las cuevas artificiales fueron desmontadas e higienizadas cada dos semanas, a la vez que los animales eran examinados en relación a su condición general y peso. Cada vez que se realizó esta tarea, el sistema se mantuvo cerrado sin permitir la interacción entre los individuos por un lapso de dos días a fin de garantizar que cada animal marcara nuevamente su cueva con orina y heces.
El nido realizado por cada individuo se mantuvo sin manipular en su lugar original. El alimento fue provisto diariamente al finalizar la sesión de observaciones y consistió en zanahorias, batatas, lechuga, choclo, alfalfa, pastos mixtos y semillas de girasol. También se colocó viruta de madera en las cámaras de crianza, de recursos y espacio de uso común. El bioterio fue mantenido bajo condiciones naturales de fotoperíodo.

\section{Muestreo}

Observaciones preliminares permitieron distinguir ritmos de actividad alternados donde se registraron períodos con una alta riqueza de interacciones sociales, seguidos por otros donde no se verificaron interacciones y cada animal se dedicó a alimentarse, descansar o autoacicalarse. Este patrón arrítmico de actividad, con asincronía entre individuos, ha sido descripto para C. talarum en cautiverio (Luna et al. 2000). Dadas estas condiciones, las sesiones de muestreo se extendieron por $1,5 \mathrm{~h}$ lo que permitió cubrir diferentes fases del comportamiento (interacción, descanso). Las sesiones fueron llevadas a cabo una o dos veces al día (10:00 y 15:00 h). El muestreo utilizado fue de tipo barrido múltiple instantáneo (Martin \& Bateson 1993), donde la ubicación en el sistema (tomada de la numeración provista a cada tramo de cada cueva individual) y comportamiento de cada individuo fue registrado a intervalos regulares de $1 \mathrm{~min}$.

La obtención de datos comenzó una semana después del ingreso de los animales al sistema de túneles, permitiendo así que cada individuo reconozca la cueva asignada como su propio territorio. Utilizando el mismo criterio, toda vez que un individuo fue reemplazado en el sistema, el mismo permaneció cerrado durante el mismo período.

El estudio se extendió por seis meses en el caso de los animales provenientes de MC y tres meses para los de $\mathrm{N}$ (incluídos dentro de la estación reproductiva de estas poblaciones), obteniéndose así 70 y 57 h totales de observación, respectivamente.

\section{Variables conductuales}

Durante los encuentros entre conespecíficos, se identificaron distintos tipos de comportamientos (Tabla 1), los cuales fueron categorizados siguiendo el criterio utilizado por Gavish et al. (1983):

(a) comportamientos individuales, que incluye actividades diarias que no implican interacción 
TABLA 1

Comportamientos identificados durante encuentros con conespecíficos en individuos de Ctenomys talarum observados en condiciones seminaturales. Ponderación: denota el peso otorgado a cada conducta agonística en el cálculo del índice de dominancia

Behaviors sampled during conspecific encounters of Ctenomys talarum individuals observed in seminatural enclosures. Weight assigned to agonistic behaviors to calculate dominance indices is shown

\begin{tabular}{|c|c|c|c|}
\hline Categoría & Tipo & Ponderación & Descripción \\
\hline Individual & & & Alimentación; descanso; locomoción; acarreo de alimento \\
\hline \multirow[t]{2}{*}{ Reconocimiento } & Olfateo & & $\begin{array}{l}\text { Toma de información por olfación del aire al entrar en una } \\
\text { cueva, directamente sobre un individuo o sus productos (ori- } \\
\text { na y heces) }\end{array}$ \\
\hline & Demarcación & & $\begin{array}{l}\text { Orinación acompañada frecuentemente del frotamiento de la } \\
\text { zona ano-genital sobre las paredes de la cueva }\end{array}$ \\
\hline \multirow[t]{11}{*}{ Agonística } & Pelea & & $\begin{array}{l}\text { Incluye un conjunto de acciones: boxeo; aprontes; muestra de } \\
\text { dientes; topeteo y mordidas }\end{array}$ \\
\hline & Con dominancia & +3 & El individuo pelea avanzando siempre hacia delante \\
\hline & Con retroceso & +2 & $\begin{array}{l}\text { El individuo pelea retrocediendo en forma permanente o } \\
\text { alternada }\end{array}$ \\
\hline & Muerde & +3 & $\begin{array}{l}\text { Un individuo profiere mordiscos a otro, quien no responde de } \\
\text { la misma manera }\end{array}$ \\
\hline & Muestra dientes & +2 & El animal levanta la cabeza quedando expuestos sus incisivos \\
\hline & Correteo & +2 & Un animal persigue a otro \\
\hline & Rechazo & +1 & $\begin{array}{l}\text { El animal realiza un apronte, frecuentemente acompañado de } \\
\text { una vocalización particular identificada }\end{array}$ \\
\hline & Vocalización tuc-tuc & +3 & $\begin{array}{l}\text { Vocalización territorial, en general utilizado luego de despla- } \\
\text { zar a un individuo de su propia cueva }\end{array}$ \\
\hline & Evitación & -2 & $\begin{array}{l}\text { Acción de desplazarse en función del no encuentro con otro } \\
\text { individuo que entra a su cueva }\end{array}$ \\
\hline & Huir a cueva ajena & -3 & $\begin{array}{l}\text { Refugiarse en otra cueva como resultado del desplazamiento } \\
\text { de la suya }\end{array}$ \\
\hline & Grupa & -1 & $\begin{array}{l}\text { Acción de darse vuelta frente a presencia de otro individuo } \\
\text { próximo a él }\end{array}$ \\
\hline
\end{tabular}

con otros individuos del sistema: alimentación, descanso, locomoción, autoacicalado en ausencia de otro conespecífico en sus proximidades; (b) comportamientos de reconocimiento, que incluye actividades involucradas en la emisión y recepción de información química entre conespecíficos, tanto mediando interacciones territoriales como de apareamiento; y (c) comportamientos agonísticos que comprende conductas que implican distinto grado de perjuicio físico de los individuos en interacción, así como la evitación del encuentro entre conespecíficos.

La manifestación de cada variable o grupo de ellas a lo largo del estudio fue promediada para cada individuo, expresándose como porcentaje (media \pm ES). La puesta a prueba de diferencias entre poblaciones y sexos para los distintos tipos de comportamientos se realizó mediante un ANOVA de dos vías, previa aplicación de la transformación arcoseno. Si bien el número muestral obtenido en este estudio es pequeño $(n=$ 6 para cada población), la normalidad y homogeneidad de varianzas que caracteriza a los datos obtenidos así como el diseño balanceado, permiten avalar la confiabilidad de los resultados estadísticos (Underwood 1997). Para cada macho de ambas localidades se calculó un índice de dominancia (ID) según la fórmula:

ID $=\Sigma$ (frecuencia de un acto $\mathrm{x}$ peso de ese acto) / $\Sigma$ frecuencia de cada acto (Barki et al. 1992).

Se asignó a cada conducta agonística un valor (peso) en una escala numérica de rango +3 (mayor agresividad) a -3 (mayor evitación); en la Tabla 1 se muestra el peso asignado a cada conducta 
agonística. Los ID calculados para cada día en que ocurrieran interacciones agonísticas entre machos de cada población se muestran como media \pm DE y fueron comparados utilizando la prueba $U$ de Mann-Whitney.

\section{RESULTADOS}

Una vez que los animales ingresaron al sistema artificial de túneles, lo demarcaron con orina y heces en toda su extensión. El nido fue construido utilizando viruta y pastos; su ubicación dentro del sistema de túneles varió entre individuos y, en algunos casos, fue cambiado a lo largo del estudio.

\section{Territorialidad individual}

Todos los individuos permanecieron la mayor parte del tiempo en su cueva individual. Aunque durante las interacciones los individuos ingresaron a cuevas ajenas, se mantuvo la pertenencia a la cueva asignada al inicio del ensayo, lo cual da validez al sistema utilizado ya que cada individuo se mantuvo solitario y fiel a una cueva individual. Sólo un $0,1 \%$ de la actividad total registrada ocurrió en el espacio común, en actitud de locomoción o exploración. El sexo, pero no la localidad de origen, afectó el tiempo de permanencia de los animales en su cueva individual $\left(\mathrm{F}_{1,11}=6,216\right.$; $\mathrm{P}=0,037$ y $\mathrm{F}_{1,11}=0,757 ; \mathrm{P}=0,409$, respectivamente). Las hembras permanecieron más tiempo en su cueva $(\mathrm{MC}=91,2 \pm 3,1 \%, \mathrm{n}=86,5 \pm 3,1 \%)$ que los machos $(\mathrm{MC}=78,4 \pm 3,1 \%, \mathrm{n}=69,5 \pm 3,1$ $\%)$. Los individuos de cada sexo no difirieron en el tiempo de permanencia en su cueva dependiendo de su localidad de origen $\left(\mathrm{F}_{1,11}=0,058 ; \mathrm{P}=\right.$ $0,815)$. El tiempo destinado a actividades individuales (Tabla 2) no fue afectado por la localidad de origen $\left(\mathrm{F}_{1,11}=0,045 ; \mathrm{P}=0,837\right)$ el sexo $\left(\mathrm{F}_{1,11}=\right.$ $4,622 ; \mathrm{P}=0,064)$, ni por la interacción entre ambos factores $\left(\mathrm{F}_{1,11}=4,180 ; \mathrm{P}=0,075\right)$.

\section{Comportamiento social}

La frecuencia de interacciones sociales (Tabla 2) no fue afectada por la localidad de origen de los individuos $\left(\mathrm{F}_{1,23}=0,003 ; \mathrm{P}=0,956\right)$. La proporción de interacciones agonísticas superó a las de reconocimiento $\left(\mathrm{F}_{1,23}=20,518, \mathrm{P}=0,0002\right)$ pero su manifestación no dependió de la localidad de origen $\left(\mathrm{F}_{1,23}=1,809 ; \mathrm{P}=0,194\right)$. Se detectaron diferencias entre sexos en el uso de conductas de reconocimiento $\left(\mathrm{F}_{1,11}=5,76 ; \mathrm{P}=0,043\right)$ donde los machos practicaron una proporción mayor de olfateos y demarcaciones con orina; sin embargo, no se verificaron diferencias entre localidades $\left(\mathrm{F}_{1,11}=1,32 ; \mathrm{P}=0,284\right)$ ni interacción entre ambos factores $\left(F_{1,11}=1,27 ; P=0,292\right)$. Diferentes modalidades de emisión y obtención de información a través de señales odoríferas fueron distinguidas en este estudio. La demarcación con orina y frotación de la zona ano-genital fue utilizada por ambos sexos durante el cortejo sexual así también como una manifestación del comportamiento territorial. El comportamiento a posteriori de un evento de olfación fue variable (Tabla 3). En el caso de las conductas agonísticas, también fueron detectadas diferencias entre sexos $\left(\mathrm{F}_{1,11}=12,89\right.$; $\mathrm{P}=0,007)$ pero no así entre localidades $\left(\mathrm{F}_{1,11}=3\right.$, $600 ; \mathrm{P}=0,094$; Tabla 2). En este caso, fue detectada interacción significativa entre sexo y locali$\operatorname{dad}\left(\mathrm{F}_{1,11}=12.76, \mathrm{P}=0.003\right)$. Para los individuos de MC existe una diferencia marcada entre sexos, siendo los machos los que manifiestan mayor cantidad de eventos agonísticos (prueba de comparaciones múltiples a posteriori de StudentNewman-Keuls, $q=7,804 ; \mathrm{P}<0,05)$, mientras que para el grupo de $\mathrm{N}$ ambos sexos se comportaron en forma similar. Las hembras de MC mostraron menor cantidad de eventos agonísticos que las hembras $(\mathrm{q}=6,112 ; \mathrm{P}<0,05)$ y los machos $(\mathrm{q}$ $=5,588 ; \mathrm{P}<0,05)$ de $\mathrm{N}$.

En la Fig. 2 se detalla la frecuencia de ocurrencia de cada tipo de interacción agonística en ambas localidades. Para MC fue posible distinguir

TABLA 2

Porcentaje de comportamientos de tipo individuales, de reconocimiento y agonísticos (media $\pm \mathrm{EE}$ ) en individuos de Ctenomys talarum interactuando en sistemas de túneles artificiales

Percentage of different kind of behaviors recorded for Ctenomys talarum individuals interacting in seminatural enclosures. Data are showed as mean $\pm \mathrm{SE}$

\begin{tabular}{lcccrrr}
\hline & \multicolumn{2}{c}{ Individuales } & \multicolumn{2}{c}{ Reconocimiento } & \multicolumn{2}{c}{ Agonísticos } \\
& Machos & Hembras & Machos & Hembras & Machos & Hembras \\
\hline Mar de Cobo & $94,9 \pm 0,4$ & $98,2 \pm 0,4$ & $1,5 \pm 0,2$ & $0,3 \pm 0,2$ & $3,7 \pm 0,2$ & $0,9 \pm 0,2$ \\
Necochea & $96,7 \pm 0,4$ & $96,8 \pm 0,4$ & $0,7 \pm 0,2$ & $0,3 \pm 0,2$ & $2,6 \pm 0,2$ & $2,9 \pm 0,2$ \\
\hline
\end{tabular}


TABLA 3

Conductas de reconocimiento (A: demarcación, B: olfación) identificadas durante encuentros con conespecíficos en individuos de Ctenomys talarum y el contexto en el que se desarrollaron

Interactions involved in recognition between individuals (A: demarcation, B: olfaction) identified during encounters with conspecifics in Ctenomys talarum. The behavioral context in which interactions were detected is provided

(A) Demarcación

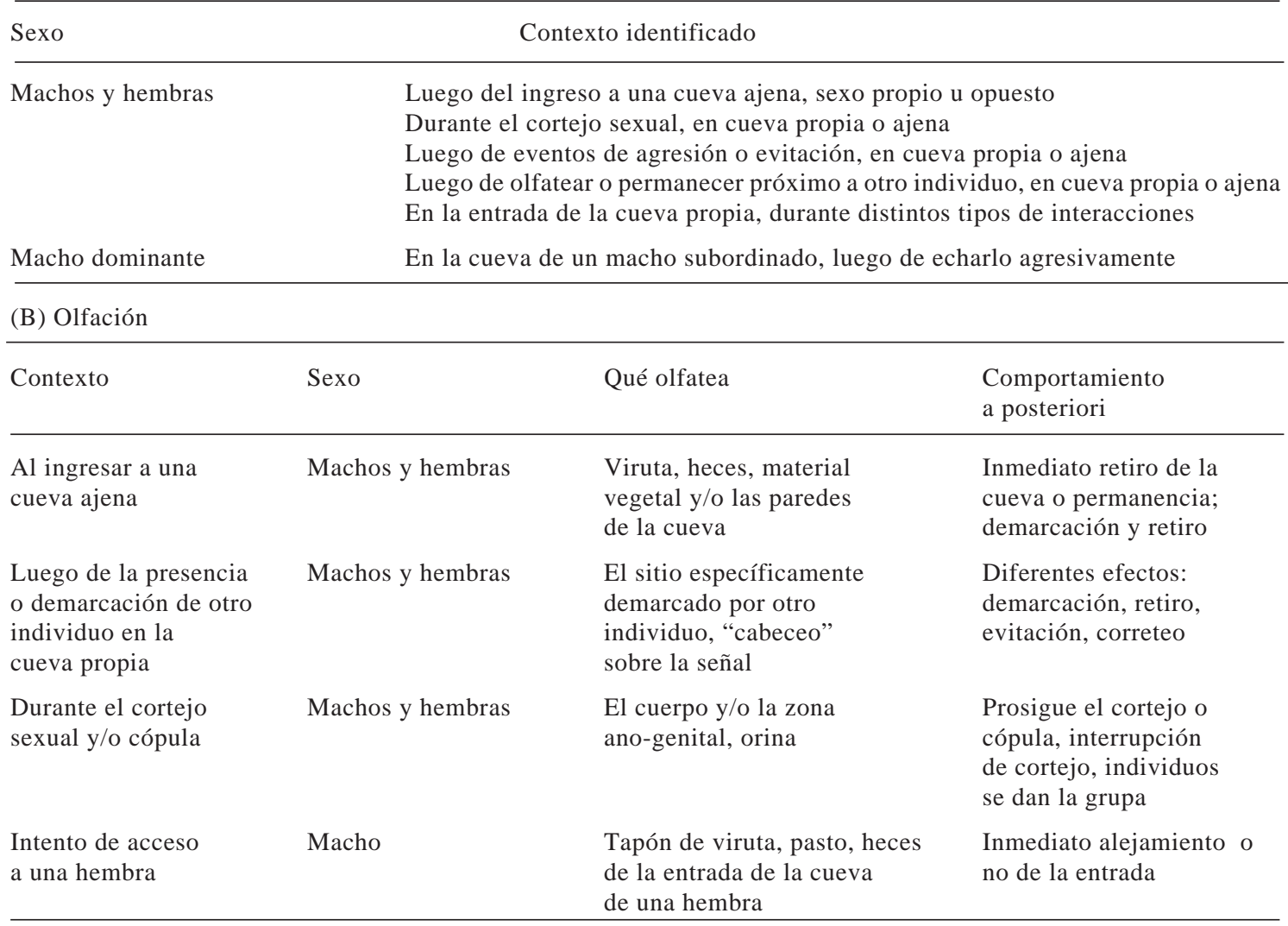

que los machos fueron los que ejercieron actitudes de mayor perjuicio ya que pelearon en mayor proporción que las hembras y fueron los únicos que mostraron vocalización territorial. Las hembras mostraron principalmente el ejercicio de actitudes vinculadas a rechazo, también evitación y mostrar la grupa. Para el caso de N, las diferencias entre sexos fueron menos marcadas: hubo registros de peleas y correteos para hembras; ambos sexos practicaron evitación, rechazo y mostrar la grupa en proporciones similares. En MC se visualizó la generación de una jerarquía de dominancia donde uno de los machos (M1) dominó las peleas mientras que los otros (M2 primero y M3 después) pelearon retrocediendo, luego lo evitaron y llegaron a ser desplazados de su propia cueva (Fig. 3). El reemplazo de M2 por M3 se debió a las heridas cortantes que el primero reci- biera y su persistente comportamiento de escape rompiendo su cueva artificial. Además, el M1 fue el único que utilizó vocalización territorial tanto en su cueva como en ajenas luego de desplazar al individuo subordinado. Esta asimetría entre machos resultó también evidente mediante el cálculo de índices de dominancia; el ID correspondiente al M1 fue superior al del M2 (2,52 $\pm 0,34 \mathrm{y}-$ $0,58 \pm 2,25$, respectivamente; $\mathrm{T}=37, \mathrm{n}_{1}=8, \mathrm{n}_{2}=$ 9, $\mathrm{P}<0,0001)$. El mismo patrón fue observado como resultado de las interacciones agonísticas entre el M1 y el M3 (2,50 $\pm 0,41$ y $-0,37 \pm 1,63$, respectivamente; $\mathrm{T}=413,5, \mathrm{n}_{1}=16, \mathrm{n}_{2}=18, \mathrm{P}<$ 0,0001). Por otro lado, en $\mathrm{N}$ no se detectaron diferencias entre los machos, las peleas predominantes fueron las denominadas «con retroceso». Ningún individuo excluyó a otro de su propia cueva y predominaron actitudes que implicaron 

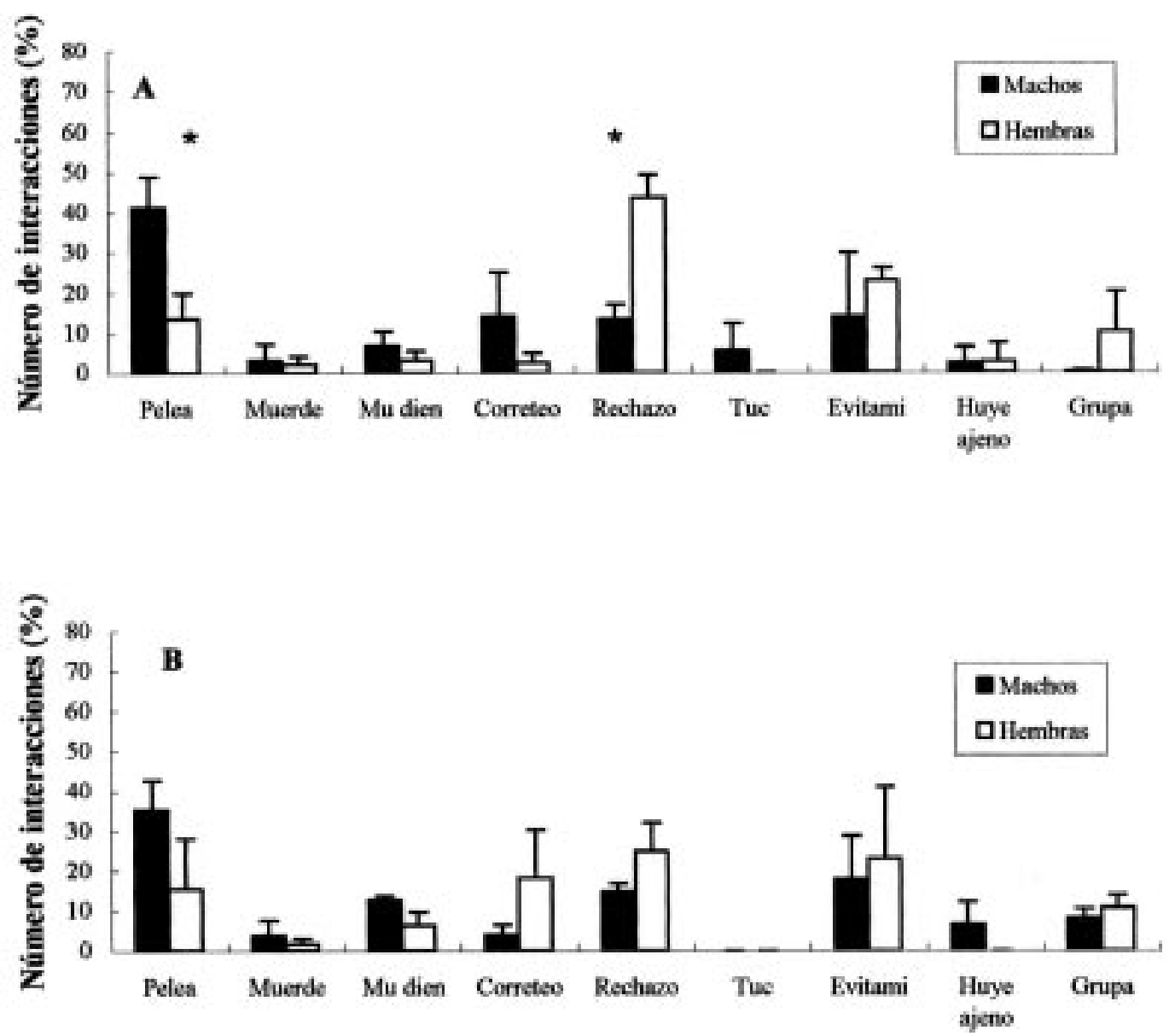

Fig. 2: Porcentaje de diferentes tipos de interacciones agonísticas registradas para individuos de Ctenomys talarum provenientes de las localidades de Mar de Cobo (A) y (B) Necochea; (*) P < 0,05 Prueba U de Mann-Whitney. Clave de abreviaciones: Mu dien: muestra dientes, tuc: vocalización territorial, evitami: evitación, huye ajeno: huye a cueva ajena.

Percentage of different types of agonistic interactions recorded for Ctenomys talarum individuals from Mar de Cobo (A) and (B) Necochea localities; (*) P < 0.05 Mann- Whitney U-test. Key to abbreviations: Pelea: fighting, muerde: biting, mu dien: showing teeth, correteo: persecution, rechazo: chasing, tuc: territorial vocalization, evitami: evading, huye ajeno: refuge in another's burrow, grupa: showing back.

menor perjuicio físico, tales como la evitación y mostrar grupa y dientes (Fig. 3). Es así como los ID obtenidos tanto para la primera pareja de machos en interacción $(\mathrm{M} 1=1 \pm 1,73, \mathrm{M} 2=0 \pm$ $2,31)$ como para la segunda $(\mathrm{M} 1=1,73 \pm 0,46$, $\mathrm{M} 3=1,22 \pm 1,36)$ no difirieron significativamente entre sí $\left(\mathrm{T}=18, \mathrm{n}_{1}=4, \mathrm{n}_{2}=5, \mathrm{P}=0,73 ; \mathrm{T}=288\right.$, $\mathrm{n}_{1}=16, \mathrm{n}_{2}=17, \mathrm{P}=0,575$, respectivamente). En el caso de MC, el establecimiento de la jerarquía de dominancia determinó la prioridad en el acceso a las hembras. El M1 cortejó y copuló con las tres hembras del sistema mientras que los subordinados (M2 y M3) nunca llegaron a iniciar un cortejo. Por otro lado, en el sistema de $\mathrm{N}$ no se generó una jerarquía que diferenciara a los machos. La cópula observada incluyó inicialmente, durante el cortejo, a los dos machos aunque finalmente uno de ellos abandonó el lugar.

\section{Descripción del comportamiento reproductivo}

El cortejo de los individuos de MC se caracterizó por la manifestación de una serie de comportamientos: una vocalización particular, sólo identificada en eventos de cortejo tanto en machos como en hembras (muy cadenciosa en machos y similar a un gemido en hembras); olfateo de zona genital, especialmente practicado por machos hacia las hembras; intento de monta por el macho, practicando mordiscos suaves en el cuello de la hembra; interrupción de monta por movimientos 
de evitación efectuados por la hembra; aproximación de la hembra en búsqueda de contacto con la pareja; el macho marca con orina en la cueva de la hembra; el macho olfatea la zona anal de la hembra o su marca de orina haciendo movimientos rítmicos con la cabeza.

Posteriormente, el comportamiento de precópula y cópula se caracterizó por la secuencia: ambos sexos despliegan vocalización de cortejo; el macho muerde el cuello de la hembra y asido de sus flancos la monta, posteriormente se detectan movimientos pélvicos; la hembra interrumpe la monta moviéndose pero se queda próxima al macho; la hembra queda con la espalda en el piso y el macho la muerde sin agresión; se repite la monta, la interrupción de la misma y los mordiscos gentiles; finalmente, el macho marca en la cueva de la hembra, se autoacicalan en proximidad, permanecen unos minutos manteniendo contacto corporal de costado; el macho se retira de la cueva y ambos prosiguen con otras actividades (alimentación, descanso).
Una vez generada la jerarquía de dominancia, el M1 copuló con las tres hembras del sistema. Con cada una de ellas practicó un número variable de cortejos anteriores y posteriores a la cópula. En todos los casos, los cortejos y las cópulas se llevaron a cabo en la cueva de la hembra cortejada. Si bien la $\mathrm{H} 3$ practicó vocalización de cortejo en proximidad del macho M2 y el M3, en ninguno de los casos hubo respuesta que implicara cortejo por alguno de ellos. En ambas situaciones, el macho M1 apartó agresivamente al macho subordinado.

A diferencia de los individuos de MC, los de $\mathrm{N}$ mostraron, durante el cortejo, menor cantidad de actitudes asociadas a vocalización o exploración de olores entre los miembros de la pareja en interacción. Previo a los intentos de monta por los machos, el cortejo resulta muy similar a una pelea. El mismo patrón se observó durante la precópula y cópula; caracterizada por topeteos y pelea con mordiscos (aunque no cortantes). La formación de una pareja no estuvo precedida por
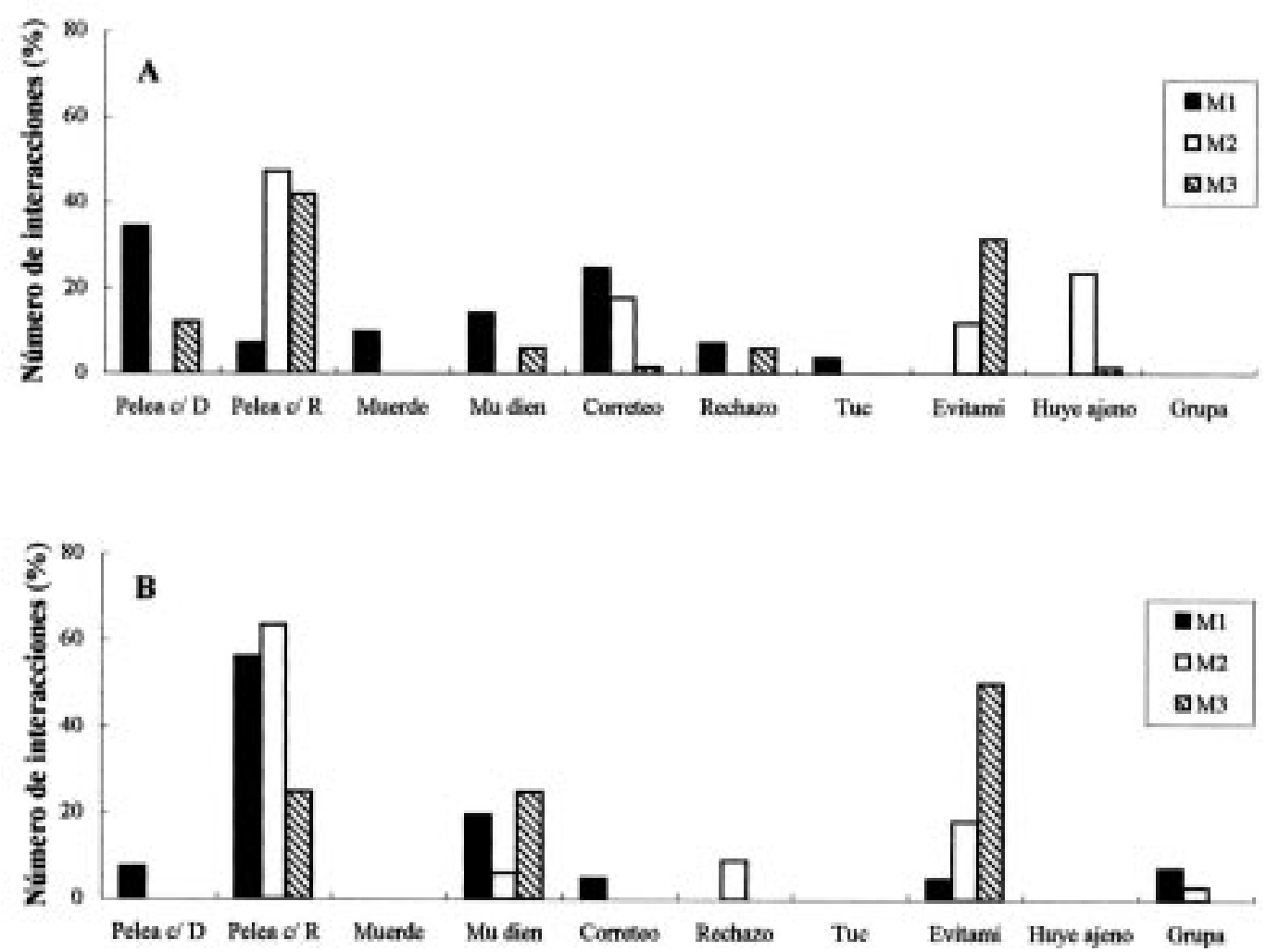

Fig. 2: Porcentaje de interacciones agonísticas que involucran solamente a machos interactuando entre sí provenientes de las localidades de Mar de Cobo (A) y Necochea; (B). Machos = M1, M2, M3. Pelea c/ D: pelea con dominancia, pelea c/R: pelea con retroceso, mu dien: meustra dientes, tuc: vocalización territorial, evitami: evitación, huye ajeno: huya a cueva ajena.

Percentage of agonistic interactions that occured among interacting males from Mar de Cobo (A) and Necochea (B) localities; Males = M1, M2, M3. Pelea c/D: fighting with dominance, pelea c/R: fighting with withdrawal, muerde: biting, mu dien: showing teeth, correteo: persecution, rechazo: chasing, tuc: territorial vocalization, evitami: evading, huya ajeno: refuge in another's burrow, grupa: showing back. 
el establecimiento de una jerarquía de dominancia entre machos. Inicialmente ambos machos participaron del cortejo sin que se manifestara agresión entre ellos. Previamente a la única cópula verificada en este grupo de individuos, la $\mathrm{H} 1$ fue cortejada repetidas veces por el macho M1 y en menor cantidad por el M2, ocurriendo esto tanto en la cueva de la hembra como en la de los machos.

\section{DISCUSIÓN}

El sistema implementado en este estudio ha proporcionado las condiciones necesarias para el mantenimiento de territorios individuales permitiendo a los tuco-tucos realizar sus actividades diarias sin interferencia con otros individuos. Además, la conexión entre los territorios individuales mediado por un espacio de uso común permitió la libre interacción entre los individuos. De esta manera, el sistema cumple con el objetivo de no forzar la interacción entre individuos, preservando el comportamiento solitario de los tucotucos tal como ocurre en la naturaleza, aumentando a la vez la probabilidad de interacción como consecuencia de la proximidad de las cuevas artificiales. El prolongado tiempo de observación necesario para analizar el comportamiento social y reproductivo de grupos de individuos en interacción, en condiciones que se procuró fueran parecidas a las naturales para la especie, limitó el número total de animales observados. Sin embargo, permitió evidenciar un variado espectro de comportamientos de difícil observación en el campo o mediante la implementación de experimentos donde sólo interactúan pares de individuos en un corto plazo.

Los tiempos de permanencia registrados en la cueva propia dan cuenta de la validez del método utilizado, reflejando el hábito solitario de esta especie en el campo (Busch et al. 1989), aún durante el período reproductivo. A diferencia de otros grupos de mamíferos que defienden áreas de uso exclusivo al mismo tiempo que presentan sobreposición en sus ámbitos de hogar, los roedores subterráneos muestran o bien superposición total de territorios - especies coloniales o sociales - o territorios exclusivos en especies solitarias (Lacey 2000). Dado que los roedores subterráneos restringen sus desplazamientos básicamente a túneles que implican un alto costo de construcción (Vleck 1979), se espera que en especies solitarias los individuos las defiendan evitando el contacto con intrusos. Sin embargo, durante la época reproductiva, cuando se registra un aumento en el grado de interacción entre indivi- duos, la territorialidad extrema podría relajarse, dando lugar a un uso compartido de túneles. Aún sin sobreposición de territorios, el acceso a varias hembras puede darse tanto por una mayor extensión de las cuevas en los machos, así como por la ubicación relativa de los sexos en el terreno. En el caso de $C$. talarum, se ha reportado que en la localidad de MC los machos ocupan áreas más extensas que las hembras, construyendo túneles más extensos y ramificados (Antinuchi \& Busch 1992). En cuanto a la disposición espacial, en las poblaciones de MC y N se ha registrado un patrón de distribución espacial agrupado donde cada macho adulto se encuentra rodeado por varias hembras (Malizia 1994).

Nuestros resultados muestran que las conductas agonísticas fueron más frecuentes que las de reconocimiento, siendo éstas últimas comúnmente utilizadas por los machos en ambas poblaciones. Esto permite proponer que la territorialidad en $C$. talarum incluye tanto el uso de señales químicas así como conductas agresivas, siendo estas últimas probablemente de mayor importancia que las primeras.

Existen diversos criterios que permiten la evaluación de jerarquías de dominancia (Dewsbury 1982). En este sentido, este estudio provee para la población de MC dos importantes fuentes de evidencia: (a) la prioridad (y exclusividad) en el acceso a hembras por uno de los machos y (b) la asimetría reflejada en los encuentros agonísticos, donde un macho (dominante) ejerce actitudes de grave perjuicio físico y emite vocalización territorial a otro (subordinado) que en primera instancia lo evita, huyendo finalmente de su propia cueva. Por el contrario, para los individuos de $\mathrm{N}$, no se verificó prioridad en el acceso a una hembra, predominaron conductas agonísticas que implicaron bajo perjuicio físico, sin evidencias de asimetría en los encuentros agresivos entre machos.

Las hembras de $C$. talarum se distribuyen en ambas poblaciones según un patrón espacial agregado (Malizia 1994), constituyendo así un recurso económicamente defendible para los machos. La proporción sexual operativa desviada hacia las hembras favorecería la monopolización múltiple de hembras en MC (mediada por la generación de una jerarquía de dominancia), mientras que en $\mathrm{N}$ una proporción sexual balanceada dificultaría este ejercicio debido al aumento en el costo de defensa de un territorio que incluya varias posibles parejas (sensu Ostfeld 1990).

Los mamíferos utilizan primariamente tres canales de comunicación: visual, químico y acústico en combinación, con énfasis en uno o más dependiendo de sus capacidades sensoriales 
(Bradbury \& Vehrencamp 1998). Uno de los problemas claves que deben resolver los organismos solitarios consiste en que deben encontrar, atraer y reconocer parejas a la vez de establecer sus límites territoriales. Se esperaría que los roedores subterráneos solitarios utilicen en gran medida las señales químicas por su condición de mamífero, por compensación de la menor relevancia del canal de comunicación visual y por ocupar un medio (suelo) altamente adecuado para la retención de este tipo de señales. Recientemente se ha confirmado que $C$. talarum posee la capacidad de distinguir señales individuales de la orina, heces y viruta conteniendo restos de estos elementos, exudados glandulares y productos de degradación de los mismos (Zenuto \& Fanjul resultados no publicados). Así, el comportamiento de almacenar restos vegetales mezclados con heces y probablemente orina en las entradas de las cuevas, verificado tanto en el campo como en cautiverio, brindaría información sobre la identidad del habitante de la madriguera.

En este estudio se ha reconocido un complejo repertorio vocal, tanto en contextos reproductivos como territoriales, asociados a comportamientos agresivos como desplazamiento y rechazo de individuos.

En las especies de Ctenomys para las cuales se describió su comportamiento copulatorio, se reportó alo-acicalamiento y contactos directos presumiblemente transmitiendo información entre posibles parejas, mientras que los mordiscos suaves probablemente provoquen apaciguamiento (Altuna et al. 1991). Además de los contactos corporales, otra manera posible de indicar intenciones reproductivas por un sexo y disminuir la agresión en el otro puede estar mediada por la vocalización. Este comportamiento fue posiblemente de importancia en los individuos de $C$. talarum de MC, mientras que no fue identificado en los de $\mathrm{N}$ (aunque no es posible descartar su uso en esta población dado que en este estudio se obtuvo un solo caso de cópula). Por otro lado, el autoacicalado de la zona genital ha sido relacionada a un rol comunicativo entre miembros de la pareja. Ferkin et al. (1996) demostraron experimentalmente en Microtus pennsylvanicus que este comportamiento permite a los machos aumentar su atracción por las hembras. La disminución de la agresión entre los miembros de la pareja mediante contactos corporales ha sido reportada por Gazit \& Terkel (2000) para S. ehrenbergi. Asimismo, Nevo (1969) atribuye el despliegue de un elaborado cortejo en esa misma especie en función de superar la hostilidad que caracteriza los encuentros entre individuos y así inducir a las hembras a copular.
Finalmente, se verificaron diferencias en el comportamiento de adquisición de pareja que se correlacionaron con las características ecológicas de estas poblaciones. En el grupo de MC, el dimorfismo sexual es más acentuado (siendo los machos de mayor tamaño que las hembras; Zenuto et al. 1999b) lo que concuerda con nuestras evidencias de un mayor grado de competencia entre machos por apareamientos y mayores niveles de agresión. En esta población, el comportamiento de cortejo y cópula estuvo fuertemente ligado a la generación de una jerarquía de dominancia entre machos. En forma contrastante, asociado al bajo potencial de defensa de múltiples hembras en la población menos densa y con equidad en el número de machos y hembras de $\mathrm{N}$, la selección sexual no premia en la misma medida un incremento en el tamaño corporal de los machos en relación a las hembras, sino un mayor tamaño relativo de testículos (Zenuto et al. 1999b) así como una mayor y más variable dotación de esperma (Zenuto 1999). Esto concuerda con la ausencia de una jerarquía de dominancia entre los machos de $\mathrm{N}$ y el cortejo plural observado en los individuos de esta población. Por otro lado, aunque el patrón general de comportamientos involucrados en el cortejo es similar para individuos de ambas poblaciones, en los individuos de MC se verificó mayor incidencia de comportamientos de cortejo complejos como vocalización, olfateo y contacto corporal, lo cual probablemente permite transmitir intenciones reproductivas al mismo tiempo de disminuir los niveles de agresión entre los sexos.

Más allá del carácter solitario de C. talarum verificado en este estudio, resulta importante destacar la riqueza de comportamientos desarrollados entre conespecíficos del mismo y distinto sexo tanto en contextos territoriales como reproductivos, utilizando múltiples canales de comunicación. Algunos de estos comportamientos son modulados bajo distintas condiciones ecológicas. Ambos aspectos permiten proponer la necesidad de considerar las interacciones entre individuos pertenecientes a especies solitarias en estudios básicos de historia de vida.

\section{AGRADECIMIENTOS}

Los autores desean agradecer a los miembros del Laboratorio de Ecofisiología, especialmente al Lic. Cristian Schleich por sus comentarios, a la Universidad Nacional de Mar del Plata y al CONICET. 


\section{LITERATURA CITADA}

ÅGREN G (1990) Sperm competition, pregnancy initiation and litter size: influence of the amount of copulatory behaviour in Mongolian gerbils, Meriones unguiculatus. Animal Behaviour 40: 417-427.

ALTUNA CA, G FRANCESCOLI \& G IZQUIERDO (1991) Copulatory pattern of Ctenomys pearsoni (Rodentia: Octodontidae) from Balneario Solís, Ururuguay. Mammalia 55: 316-318.

ANTINUCHI CD \& C BUSCH (1992) Burrow structure in the subterranean rodent Ctenomys talarum. Zeitschrift für Säugertierkunde 57: 163-168.

BAKER RR \& MA BELLIS (1989) Number of sperm in human ejaculates varies in accordance with sperm competition theory. Animal Behaviour 37: 867-869.

BANDOLI JH (1987) Activity and plural occupancy of burrows in Botta's pocket gopher Thomomys bottae. American Midland Naturalist 118: 10-14.

BARKI A, I KARPLUS \& M GOREM (1992) Effects of size and morphotype on dominance hierarchies and resource competition in the freshwater prawn Macrobrachium rosenbergii. Animal Behaviour 44: 547-555.

BORRONI A, A LOY \& E CAPANNA (1999) A flexible arrangement for the study of moles in captivity. Acta Theriologica 44: 204-214.

BRADBURY JW \& SL VEHRENCAMP (1998) Principles of animal communication. Sinauer Associates, Inc., Sunderland, Massachusetts. $882 \mathrm{pp}$.

BUSCH C, AI MALIZIA, OA SCAGLIA \& OA REIG (1989) Spatial distribution and attributes of a population of Ctenomys talarum (Rodentia: Octodontidae). Journal of Mammalogy 70: 204-208.

BUSCH C, CD ANTINUCHI, JC DEL VALLE, MJ KITTLEIN, AI MALIZIA, AI VASSALLO \& RR ZENUTO (2000) Population ecology of subterranean rodents. En: Lacey EA, JL Patton \& GN Cameron (eds) Life underground: the biology of subterranean rodents: 183-226. The University of Chicago Press, Chicago, Illinois.

DALY JC \& JM PATTON (1990) Dispersal, gene flow, and allelic diversity between local populations of Thomomys bottae pocket gophers in the coastal ranges of California. Evolution 44: 1283-1294.

DEWSBURY DA (1982) Dominance rank, copulatory behavior, and differential reproduction. The Quarterly Review of Biology 57: 135-159.

EMLEN ST \& LW ORING (1977) Ecology, sexual selection, and the evolution of mating systems. Science 197: 215-223.

FERKIN MH, ES SOROKIN \& RE JHONSTON (1996) Self-grooming as a sexually dimorphic communicative behaviour in meadow voles Microtus pennsylvanicus. Animal Behaviour 51: 801-810.

GAVISH L, CS CARTER \& LL GETZ (1983) Male- female interactions in prairie voles. Animal Behaviour 31: 511-517.

GAZIT I, U SHANAS \& J TERKEL (1996) First succesful breeding of the blind mole rat (Spalax ehrenbergi) in captivity. Israel Journal of Zoology 42: 3-13.
GAZIT I \& J TERKEL (1998) Chronological development of behavior in the blind mole rat (Spalax ehrenbergi). International Journal of Comparative Psychology 11: $1-16$.

GAZIT I \& J TERKEL (2000) Reproductive behavior of the blind mole-rat (Spalax ehrenbergi) in a seminatural burrow system. Canadian Journal of Zoology 78: 570-577.

HANSEN RM \& RS MILLAR (1959) Observations on the plural occupancy of pocket gopher burrow systems. Journal of Mammalogy 40: 577-584.

KENAGY GJ \& SC TROMBULAK (1986) Size and function of mammalian testes in relation to body size. Journal of Mammalogy 67: 1-22.

LACEY EA (2000) The spatial and social systems of subterranean rodents. En: Lacey EA, JL Patton \& GN Cameron (eds) Life underground: the biology of subterranean rodents: 257-296. The University of Chicago Press, Chicago, Illinois.

LACEY EA, SH BRAUDE \& JR WIECZOREK (1997) Burrow sharing by colonial tuco- tucos (Ctenomys sociabilis). Journal of Mammalogy 78: 556-562.

LACEY EA, SH BRAUDE \& JR WIECZOREK (1998) Solitary burrow use by adult Patagonian tuco-tucos (Ctenomys haigi). Journal of Mammalogy 79: 986991.

LUNA F, CD ANTINUCHI \& C BUSCH (2000) Ritmos de actividad locomotora y uso de las cuevas en condiciones seminaturales en Ctenomys talarum (Rodentia: Octodontidae). Revista Chilena de Historia Natural 73: 39-46.

MALIZIA AI (1994) Ecología poblacional de Ctenomys talarum. Tesis Doctoral, Universidad Nacional de Mar del Plata, Mar del Plata, Argentina. 96 pp.

MALIZIA AI \& C BUSCH (1997) Breeding biology of the fossorial rodent Ctenomys talarum (Rodentia: Octodontidae). Journal of Zoology (London) 242: 463-471.

MALIZIA AI, RR ZENUTO \& C BUSCH (1995) Demographic and reproductive attributes of dispersers in two populations of the subterranean rodent Ctenomys talarum (tuco- tuco). Canadian Journal of Zoology 73: 732-738.

MARTIN P \& P BATESON (1993) Measuring behaviour: an introductory guide. Cambridge University Press, Cambridge, United Kingdom. 222 pp.

NEVO E (1969) Mole rat Spalax ehrenbergi: mating behavior and its evolutionary significance. Science 163: 484-486.

NEVO E (1979) Adaptive convergence and divergence of subterranean mammals. Annual Review of Ecology and Systematics 10: 269-308.

OSTFELD RS (1990) The ecology of territoriality in small mammals. Trends in Ecology \& Evolution 5: 411415.

PATTON JL \& JH FEDER (1981) Microspatial genetic heterogeneity in pocket gophers: non random mating and drift. Evolution 35: 912-920.

PATTON JL \& MF SMITH (1993) Molecular evidence for mating asymmetry and female choice in in a pocket gopher (Thomomys) hybrid zone. Molecular Ecology 2: $3-8$. 
SHANAS U, G HETH, E NEVO, R SHALGI \& J TERKEL (1995) Reproductive behaviour in the female blind mole rat (Spalax ehrenbergi). Journal of Zoology (London) 237: 195-210.

SHORT RV (1977) Sexual selection and the descent of man. En: Calaby JH \& CH Tyndale-Biscoe (eds) Reproduction and evolution, proceedings of the fourth symposium on comparative biology of reproduction: 3-19. Australian Academy of Science, Canberra, Australia.

UNDERWOOD AJ (1997) Experiments in ecology. Cambridge University Press, Cambridge, United Kingdom. 504 pp.

VAUGHAN TA (1962) Reproduction in the plains pocket gopher on Colorado. Journal of Mammalogy 43: 113.

VLECK D (1979) The energy cost of burrowing by the pocket gopher Thomomys bottae. Physiological Zoology 52: 122-134.

ZENUTO RR (1999) Sistema de apareamiento en el roedor subterráneo Ctenomys talarum (Rodentia: Octodontidae). Tesis Doctoral, Universidad Nacional de Mar del Plata, Mar del Plata, Argentina. 103 pp.
ZENUTO RR \& C BUSCH (1998) Population biology of the subterranean rodent Ctenomys australis (tucotuco) in a coastal dunefield in Argentina. Zeitschrift für Säugertierkunde 63: 351- 367.

ZENUTO RR, EA LACEY \& C BUSCH (1999a) DNA fingerprinting reveals polygyny in the subterranean rodent Ctenomys talarum. Molecular Ecology 8: 15291532.

ZENUTO RR, AI MALIZIA \& C BUSCH (1999b) Sexual size dimorphism, relative testes size and mating system in two populations of Ctenomys talarum (Rodentia: Octodontidae). Journal of Natural History 33: 305-314

ZENUTO RR, AI VASSALLO \& C BUSCH (2001) A method for studying social and reproductive behavior of subterranean rodents in captivity. Acta Theriologica 46: 161-170.

ZURI I \& J TERKEL (1996) Locomotor patterns, territory, and tunnel utilization in the mole-rat Spalax ehrenbergi. Journal of Zoology (London) 240: 123140.

Editor Asociado: F. Bozinovic

Recibido el 1 de octubre de 2001; aceptado el 21 de enero de 2002 